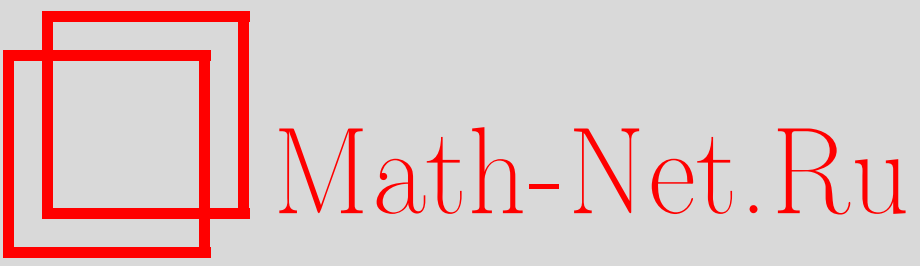

В. Г. Фокин, В. А. Дмитриев, Определение методом конечных элементов дополнительных остаточных напряжений при разрезке деталей, Вестн. Сам. гос. техн. ун-та. Сер. Физ.-мат. науки, 2009, выпуск 1(), 95-100

DOI: https://doi.org/10.14498/vsgtu661

Использование Общероссийского математического портала Math-Net.Ru подразумевает, что вы прочитали и согласны с пользовательским соглашением

http: //www. mathnet.ru/rus/agreement

Параметры загрузки:

IP : 34.227 .88 .159

26 апреля 2023 г., 18:30:54 
УДК 539.219.2:539.3

\section{ОПРЕДЕЛЕНИЕ МЕТОДОМ КОНЕЧНЫХ ЭЛЕМЕНТОВ ДОПОЛНИТЕЛЬНЫХ ОСТАТОЧНЫХ НАПРЯЖЕНИЙ ПРИ РАЗРЕЗКЕ ДЕТАЛЕЙ}

\section{В.Г. Фокин, В. А. Дмитриев}

Самарский государственный технический университет, 443100, Самара, ул. Молодогвардейская, 244.

E-mails: mechsamgtu@samgtu.ru, tms@samgtu.ru

Предлагается алгоритм использования МКЭ для определения дополнительных остаточных напряжений в кольцах со сложной бормой сечения после их разрезки. Приведен пример реализации этого алгоритма. Принятые допущения проверены путем моделирования процесса образования в кольцах типичного поля остаточных напряжений.

Ключевые слова: метод конечных элементов, остаточные напряжения, колъцо, подшипник, разрезка.

Остаточные напряжения в поверхностных слоях деталей машин оказывают значительное влияние на надежность [1]. Поэтому желательно на этапе финишных технологических операций создавать в наружных слоях ответственных рабочих поверхностей деталей сжимающие остаточные напряжения. Это условие предъявляется, например, к лопаткам компрессоров, валам, к дорожкам качения колец подшипников и другим деталям.

Теоретически рассчитать остаточные напряжения, учитывая историю получения детали, во многих случаях затруднительно из-за сложности физических и технологических процессов. Поэтому широко применяются расчётноэкспериментальные механические методы определения остаточных напряжений в поверхностных слоях, основанные на соответствующей разрезке детали с последующим травлением или обтачиванием (растачиванием) исследуемых поверхностей [1]. После разрезки измеряются деформации или перемещения образцов, возникающие вследствие разгрузки от остаточных напряжений. По измеренным величинам вычисляются дополнительные остаточные напряжения, вносимые в образцы при разрезке. Затем травлением или обтачиванием (растачиванием) последовательно удаляются наружные слои с исследуемых поверхностей образцов и измеряются возникающие при этом перемещения или деформации. На основе последних измерений вычисляются конечные остаточные напряжения в образцах. Вычитая из этих величин дополнительные напряжения, находят значения исходных остаточных напряжений в деталях. Необходимые расчётные соотношения выводятся методами сопротивления материалов или теории упругости. При этом обычно допускаются упрощения как в геометрии деталей и образцов, так и в модели их деформирования, что может заметно влиять на результаты исследований.

Метод конечных элементов (МKЭ) [2] с использованием программных средств компьютерного инженерного анализа позволяет значительно облег-

Фокин Владимир Григорьевич-дочент кафедры механики; к.т.н., дочент.

Дмитриев Владимир Александрович-дочент кафедрь технологий машиностроения; к.т.н., дочент. 
чить и уточнить расчёты остаточных напряжений при измерении их механическими методами. Рассмотрим алгоритм применения МKЭ для определения дополнительных остаточных напряжений в случае разрезки кольца роликового конического подшипника с целью измерения остаточных напряжений в поверхностных слоях дорожки качения.

Кольцо имеет достаточно сложную форму радиального сечения. После его разрезки измеряются расхождение сечений $\Delta$ и угол их взаимного поворота $\theta$ (рис. 1). Предполагается, что разгрузка остаточных напряжений в сечении разреза приводится только к паре сил, вектор-момент которой $\vec{M}=\vec{M}_{z}+\vec{M}_{y}$ лежит в плоскости сечения. Учитывая симметрию, расчёты МКЭ удобно выполнять только на половине кольца, считая нижнее сечение защемленным (рис. 2). Полагая разгрузку при разрезке кольца упругой, можно записать

$$
M_{z}=K_{1} \Delta+K_{2} \theta, \quad M_{y}=K_{3} \Delta+K_{4} \theta .
$$

Произведя на конечно-элементной модели полукольца два упругих расчёта для различных сочетаний моментов $\left(M_{z_{1}}, M_{y_{1}}\right),\left(M_{z_{2}}, M_{y_{2}}\right)$ и определив два соответствующих набора перемещений $\left(\Delta_{1}, \theta_{1}\right),\left(\Delta_{2}, \theta_{2}\right)$, из уравнений $(1)$ легко вычислить коэффициенты $K_{i}$ :

$$
\begin{aligned}
& K_{1}=\frac{M_{z 1} \theta_{2}-M_{z 2} \theta_{1}}{\Delta_{1} \theta_{2}-\Delta_{2} \theta_{1}}, \quad K_{2}=\frac{M_{z 1} \Delta_{2}-M_{z 2} \Delta_{1}}{\theta_{1} \Delta_{2}-\theta_{2} \Delta_{1}}, \\
& K_{3}=\frac{M_{y 1} \theta_{2}-M_{y 2} \theta_{1}}{\Delta_{1} \theta_{2}-\Delta_{2} \theta_{1}}, \quad K_{4}=\frac{M_{y 1} \Delta_{2}-M_{y 2} \Delta_{1}}{\theta_{1} \Delta_{2}-\theta_{2} \Delta_{1}} .
\end{aligned}
$$

Зная коэффициенты $K_{i}$ для данного типоразмера кольца, через измеренные перемещения $\Delta$ и $\theta$, полученные при разрезке кольца, по формулам (1) можно

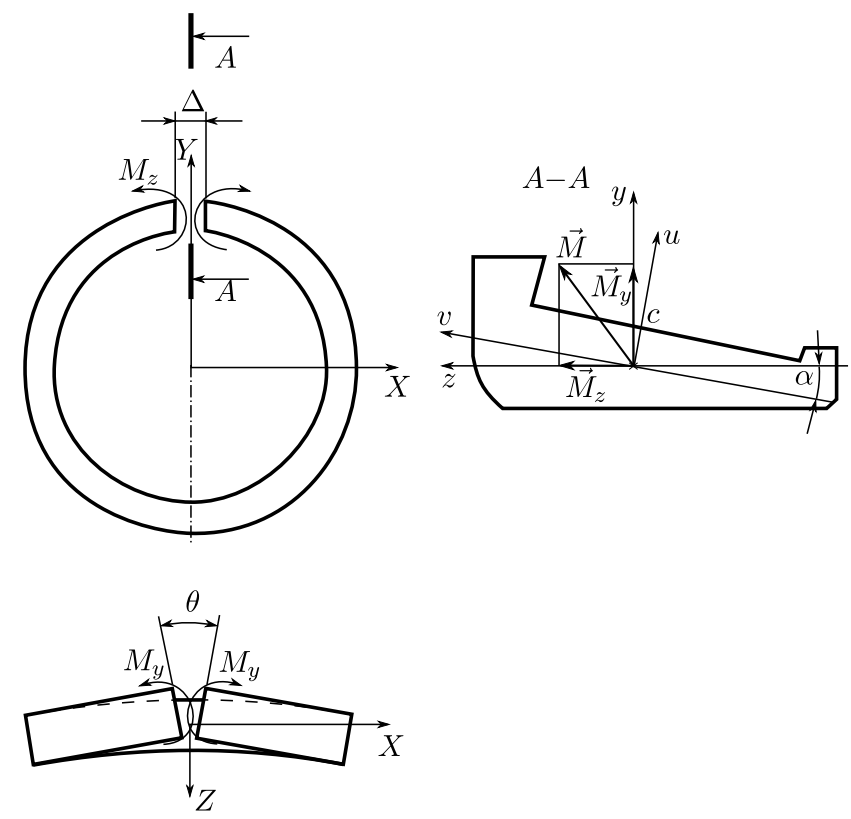

Рис. 1. Перемещения кольца при разрезке 
вычислить моменты $M_{z}, M_{y}$. Принимая в качестве нагрузки эти моменты, по расчётной схеме, показанной на рис. 2, MKЭ вычисляются дополнительные остаточные напряжения.

Изложенным выше способом были определены дополнительные окружные остаточные напряжения во внутреннем кольце конического роликоподшипника (рис. 3), характеристики которого и величины перемещений сечений при разрезке представлены в таблице. Коническая поверхность дорожки качения обработана шлифованием. Реальное сечение кольца аппроксимировалось четырехугольником. Взаимный угол поворота сечений $\theta$ был незначительным, и им пренебрегли.

Характеристики сечения кольца и перемещения при разрезке

\begin{tabular}{c|c|c|c|c|c|c|c}
\hline Тип кольца & $R, \mathrm{Mм}$ & $I_{u}, \mathrm{MM}^{4}$ & $I_{v}, \mathrm{Mм}^{4}$ & $\alpha$, град. & $U_{c}, \mathrm{Mм}$ & $\Delta$, мм & $\Theta$, град. \\
\hline $7517-02$ & 47,12 & 33200,89 & 2890,39 & 10,21 & 3,38 & 0,10 & 0 \\
\hline
\end{tabular}

В таблице: $R$-радиус окружности, проходящей через центры тяжести радиальных сечений кольца; $I_{u}, I_{v}$ - моменты инерции сечения относительно главных центральных осей; $\alpha$ - угол наклона главных центральных осей; $U_{c}$ - координата точки $c$ (см. рис. 3 ).

Предварительные расчёты МКЭ позволили найти по формулам (2) коэффициенты жесткости кольца: $K_{1}=3,303 \cdot 10^{4} \mathrm{H} ; K_{2}=-8,65 \cdot 10^{4} \mathrm{H} \cdot \mathrm{мm}$; $K_{3}=20,117 \mathrm{H} ; K_{4}=3,35 \cdot 10^{6} \mathrm{H} \cdot$ мм. Для измеренных при разрезке кольца перемещений $\Delta=0,1$ мм и $\theta \approx 0^{\circ}$ по формулам (1) вычисляются моменты,

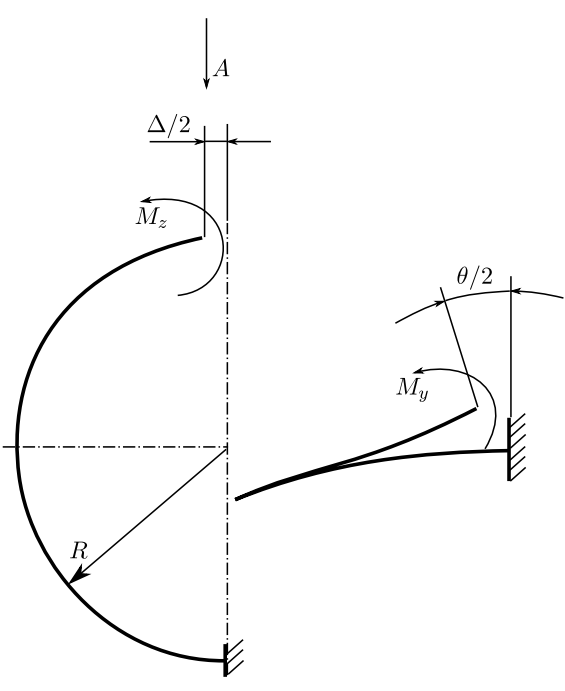

Рис. 2. Расчётная схема
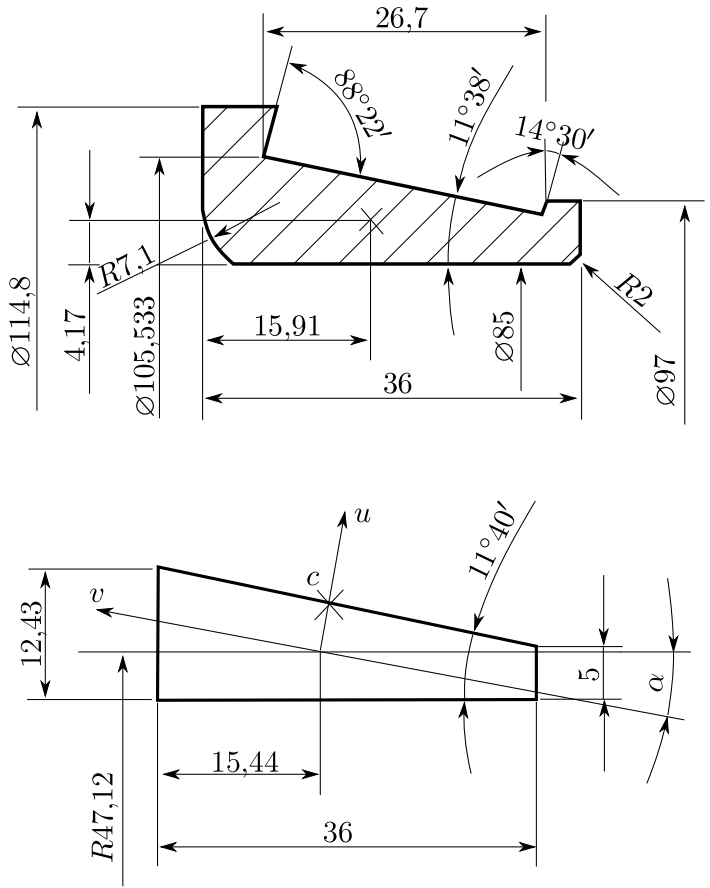

Рис. 3. Реальное и аппроксимированное сечения кольца подшипника 7517-02 
создаваемые остаточными напряжениями, действующими в радиальном сечении: $M_{z}=K_{1} \cdot \Delta=3,303 \cdot 10^{3} \mathrm{H} \cdot \mathrm{mм} ; M_{y} \approx 0$. Расчёт МКӨ кольца по схеме, представленной на рис. 2 , при нагрузке $M_{z}=3,303 \cdot 10^{3} \mathrm{H} \cdot \mathrm{мм}$ дал поле искомых дополнительных окружных остаточных напряжений, не изменяющееся в окружном направлении и переменное в осевом направлении. На поверхности дорожки качения эти напряжения изменяются от -2,9 МПа вблизи тонкого торца до $-8,7$ МПа вблизи толстого торца. Для точки $с$ в центральной зоне дорожки (см. рис. 3) дополнительное окружное напряжение оказалось равным $\sigma \approx-5 \mathrm{MПа,} \mathrm{что} \mathrm{согласуется} \mathrm{с} \mathrm{расчётным} \mathrm{значением,} \mathrm{полученным}$ по приближенной формуле, взятой из статьи [3]:

$$
\sigma \approx-\frac{E \Delta U_{c}}{2 \pi R^{2} \cos \alpha}=-\frac{2 \cdot 10^{5} \cdot 0,1 \cdot 3,38}{2 \cdot 3,14 \cdot 47,12^{2} \cdot \cos 10,21}=-4,9[\mathrm{M \Pi а}] .
$$

Здесь $E$ - модуль упругости материала кольца, другие величины даны на рис. 3 и в таблице.

В [3] для подобных колец с помощью интеграла Мора выведены расчётные формулы, позволяющие вычислять окружные дополнительные остаточные напряжения через измеренные величины $\Delta$ и $\theta$. Расчеты по этим формулам весьма трудоемки, так как требуется определять центр тяжести сложного сечения кольца, положение главных осей, его главные моменты инерции и рассчитывать значения остаточных напряжений во многих точках. Предлагаемый подход в случае использования программы, реализующей MKЭ, значительно облегчает анализ дополнительных остаточных напряжений.

Рассмотренная выше методика определения дополнительных остаточных напряжений в кольцах, так же как методика в работе [3], основаны на допущении, что остаточные напряжения в радиальном сечении кольца приводятся только к паре сил с моментом $\vec{M}=\vec{M}_{z}+\vec{M}_{y}$. Докажем МКЭ, что это условие соответствует реальной картине распределения остаточных напряжений в кольцах подшипников после типичных финишных технологических операций. Кроме того, на примере кольца (см. рис. 1) покажем допустимость использования предлагаемой методики в случае, когда ширина кольца соизмерима с радиусом.

При точении, шлифовании, дробеструйной обработке в поверхностном слое дорожки качения кольца подшипника возникают пластические деформации, которые после разгрузки приводят к образованию остаточных напряжений. Имитируем подобный физический процесс в кольце (см. рис. 1) путем внесения в поверхностный слой толщиной 0,4 мм начальных деформаций $\varepsilon_{x}=\varepsilon_{y}=\varepsilon_{z}=2 \cdot 10^{-3}$. Результаты расчёта МКЭ такого деформирования кольца даны на рис. 4.

Касательные и радиальные остаточные напряжения близки к нулю, поэтому не представлены на этом рисунке. Вне краевых зон остаточные напряжения не изменяются вдоль образующей и имеют характерное распределение по радиусу. В радиальных сечениях кольца окружные остаточные напряжения приводятся только к паре с моментом $M_{z}$. Осевые остаточные напряжения создают равномерно распределённые по окружности кольца изгибающие моменты $M_{x}$.

Полученная картина остаточных напряжений в радиальном сечении соответствует предположению о приведении их к паре сил, заложенному в ме- 
тодике определения дополнительных остаточных напряжений в кольцах. Из результатов моделирования также следует, что в поверхностном слое кольца вследствие пластических деформаций может создаваться плоское остаточное напряженное состояние, измерение которого требует дополнительных исследований на образцах, вырезанных из кольца в осевом направлении.
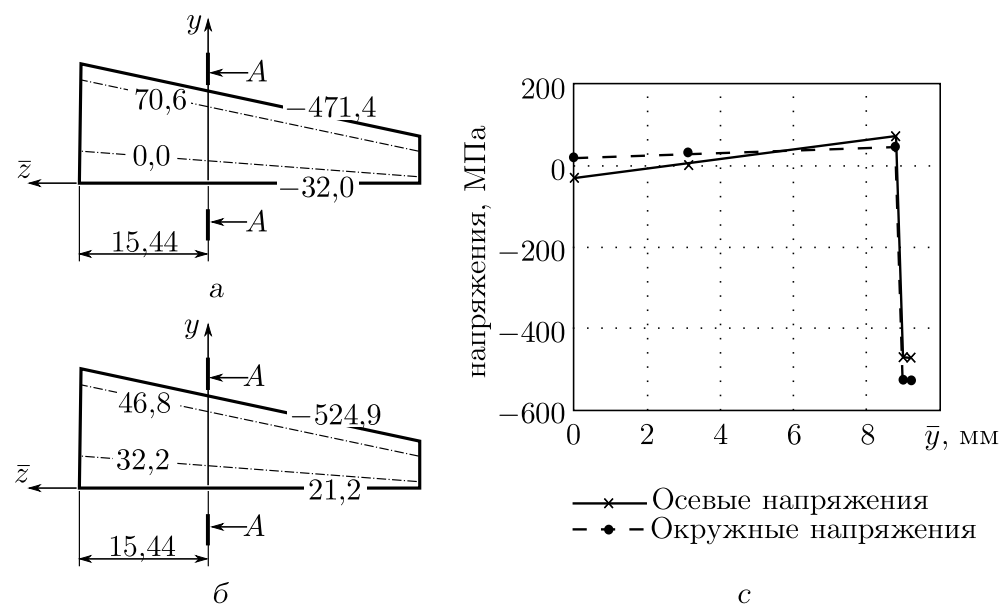

Рис. 4. Остаточные напряжения от начальных деформаций: а - изолинии осевых напряжений $\left(\sigma_{z}, \mathrm{MПа);} \mathrm{б-} \mathrm{изолинии} \mathrm{окружных} \mathrm{напряжений}\left(\sigma_{x}, \mathrm{MПа}\right) ; c-\right.$ распределение напряжений в разрезе $A-A$

Моделирование МКЭ разрезанного кольца, имеющего форму сечения как на рис. 3, с внесёнными в поверхностный конический слой начальными деформациями $\varepsilon_{x}=\varepsilon_{y}=\varepsilon_{z}=2 \cdot 10^{-3}$, показало, что в основном происходит расхождение сечений $\Delta$, а угол их взаимного поворота $\theta$ почти нулевой (рис. 5). Это совпадает с наблюдаемыми перемещениями при разрезке реального кольца роликового конического подшипника, имеющего шлифованную дорожку качения (см. таблицу).

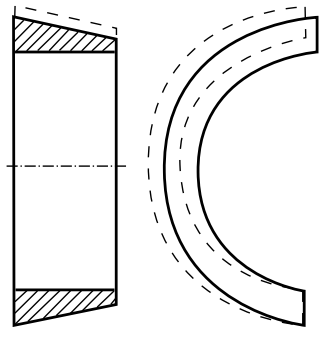

Рис. 5. Перемещения разрезанного кольца от начальных деформаций

Выводы. В работе представлен алгоритм расчётов МКЭ дополнительных остаточных напряжений в кольце подшипника по результатам измерения перемещений при разрезке. МКЭ облегчает расчёты дополнительных остаточных напряжений и повышает точность анализа в случае сложной формы радиального сечения кольца.

Предложенным методом определены дополнительные остаточные напряжения после разрезки кольца серийного подшипника со шлифованной дорожкой качения.

Моделирование MKЭ остаточных напряжений в кольце со сложным сечением, в поверхностный слой которого внесены начальные деформации, показало достоверность принятых допущений.

Разработанный алгоритм использования MKЭ в расчётах дополнительных остаточных напряжений после разрезки применим не только для колец, но и для других типов деталей, например, стержней, пластин, оболочек. 


\section{БИБЛИОГРАФИЧЕСКИЙ СПИСОК}

1. Биргер И. А. Остаточные напряжения. - М.: Машгиз, 1963. - 232 с.

2. Зенкевич О. Метод конечных элементов в технике. - М.: Мир, 1975. - 541 с.

3. Фокин В. Г. Дмитриев В.А. Определение дополнительных напряжений при разрезке кольца с остаточными напряжениями // Вестн. Сам. гос. техн. ун-та. Сер. Техн. науки, 2001. - № 11. - С. 93-96.

MSC: 74A10, 74S05

\section{DEFINITION OF ADDITIONAL RESIDUAL STRESSES OF CUT PARTS BY FINITE ELEMENTS METHOD}

\section{G. Fokin, V.A. Dmitriev}

Samara State Technical University,

244, Molodogvardeyskaya str., Samara, 443100.

E-mails: mechsamgtu@samgtu.ru, tms@samgtu.ru

Algorithm of the method of finite elements is proposed for definition of additional residual stress in rings with complex cut surface configuration. The example of algorithm application is demonstrated. Accepted assumptions are verified by modeling of the process of typical residual stress field formation in rings.

Key words: finite elements method, residual stresses, ring, bearing, cut.

Original article submitted 13/I/2009; revision submitted $02 / \mathrm{III} / 2009$.

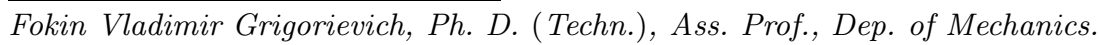
Dmitriev Vladimir Alexandrovich, Ph. D. (Techn.), Ass. Prof., Dept. of Production Engineering. 